HEAT TRANSFER - ASIAN RESEARCH

EDITOR-IN-CHIEF: PROFESSOR William. M. Worek -Texas A\&M University-Kingsville, Texas, USA.

PUBLISHER- WILEY USA

ONLINE ISSN: 1523-1496

Accepted October $9^{\text {th }} 2018$

\title{
ADOMIAN DECOMPOSITION SOLUTION FOR PROPULSION OF DISSIPATIVE MAGNETIC JEFFREY BIOFLUID IN A CILIATED CHANNEL CONTAINING A POROUS MEDIUM WITH FORCED CONVECTION HEAT TRANSFER
}

\author{
N. Manzoor ${ }^{1}$ K. Maqbool1*, 0 Anwar Bég² and S. Shaheen ${ }^{1}$ \\ 1 Department of Mathematics \& Statistics, International Islamic University, Islamabad 44000, Pakistan. \\ 2 Fluid Mechanics, Propulsion and Nano-Systems, Aeronautical and Mechanical Engineering Department, \\ G77, Newton Building, University of Salford, Manchester, M54WT, UK \\ *Corresponding author-Email: khadija.maqbool@iiu.edu.pk
}

\begin{abstract}
Physiological transport phenomena often feature ciliated internal walls. Heat, momentum and multi-species mass transfer may arise and additionally non-Newtonian biofluid characteristics are common in smaller vessels. Blood (containing hemoglobin) or other physiological fluids containing ionic constituents in the human body respond to magnetic body forces when subjected to external (extracorporeal) magnetic fields. Inspired by such applications, in the present work we consider the forced convective flow of an electrically-conducting viscoelastic physiological fluid through a ciliated channel under the action of a transverse magnetic field. The flow is generated by a metachronal wave formed by the tips of cilia which move to and fro in a synchronized fashion. The presence of deposits (fats, cholesterol etc) in the channel is mimicked with a Darcy porous medium drag force model. The two-dimensional unsteady momentum equation and energy equation are simplified with a stream function and small Reynolds' number approximation. The effect of energy loss is simulated via the inclusion of viscous dissipation in the energy conservation (heat) equation. The non-dimensional, transformed moving boundary value problem is solved with appropriate wall conditions via the semi-numerical Adomian decomposition method (ADM). The velocity, temperature and pressure distribution are computed in the form of infinite series constructed by ADM and numerically evaluated in a symbolic software (MATHEMATICA). Streamline distributions are also presented. The influence of Hartmann number (magnetic parameter), Jeffrey first and second viscoelastic parameters, permeability parameter (modified Darcy number), and Brinkman number (viscous heating parameter) on velocity, temperature, pressure gradient and bolus dynamics is visualized graphically. The flow is decelerated with increasing with increasing Hartmann number and Jeffery first parameter in the core flow whereas it is accelerated in the vicinity of the walls. Increasing permeability and Jeffery second parameter are observed to accelerate the core flow and decelerate the peripheral flow near the ciliated walls. Increasing Hartmann number elevates pressure gradient whereas it is reduced with permeability parameter. Temperatures are elevated with increasing magnetic parameter, Brinkman number and Jeffery second parameter. Increasing magnetic field is also observed to reduce the quantity of trapped boluses. Increasing permeability parameter suppresses streamline amplitudes. Both the magnitude and quantity of trapped boluses is elevated with an increase in both first and second Jeffery parameters.
\end{abstract}

KEYWORDS: Forced convection, Jeffrey model, Ciliary movement, Viscous dissipation, Adomian decomposition method (ADM), magnetohydrodynamics, physiological propulsion. 


\section{NOMENCLATURE}

\begin{tabular}{|c|c|}
\hline $\mathbf{V}$ & Velocity field vector \\
\hline$u, v$ & Horizontal and vertical velocity in wave frame \\
\hline$x, y$ & Rectangular coordinates of wave frame \\
\hline$U, V$ & Horizontal and vertical velocity in fixed frame \\
\hline$X, Y$ & Rectangular coordinates of fixed frame \\
\hline $\mathbf{J}$ & Current density \\
\hline B & Magnetic field \\
\hline$R$ & Darcy's resistance \\
\hline$P$ & Pressure \\
\hline$\theta$ & Dimensionless temperature \\
\hline$\psi$ & Stream function \\
\hline$\gamma$ & Shear rate \\
\hline$c$ & Wave speed \\
\hline$k$ & Permeability of porous medium \\
\hline$M$ & Hartmann number \\
\hline$a$ & Wave amplitude \\
\hline$\varepsilon$ & Cilia length \\
\hline$\alpha$ & Eccentricity of elliptical pair of cilia \\
\hline$\beta$ & Wave number \\
\hline$\lambda_{1}, \lambda_{2}$ & Jeffrey first and second viscoelastic parameters \\
\hline$B r$ & Brinkman number \\
\hline
\end{tabular}

\section{INTRODUCTION}

Thermal analysis of biological systems is a vibrant area of modern biomechanics and biomedical engineering. Thermal science deals with properties of the substances associated with pressure, density, velocity, viscosity and temperature and their relationship with energy. Thermal science is divided into biological thermodynamics, classical thermodynamics, equilibrium and nonequilibrium thermodynamics, statistical thermodynamics and heat transfer. The human body may be considered as an open system (heat engine). Energy conservation is based upon the 
thermodynamics laws and the fluid mechanics laws (momentum and mass conservation). The fundamental law employed in thermal conduction heat transfer is Fourier's law. Heat regulation is essential to all mammals and furthermore thermal analysis has found many exciting new applications in modern biomedical engineering. These include thermal treatment of tumours [1], heat flow in blood [2], perspiration [3], combustion processes associated with food [4], heat transfer in eye treatment [5], heat diffusion in swallowing dynamics [6], respiratory thermal control [7], cardiovascular transport [8], thermal trauma simulation and therapy [9], thermal bioconvection [10], skin burns [11], tissue thermo-mechanics [12] and thermal comfort [13]. Other applications of heat transfer in biological systems include laser radiation of tissue, preservation of biological material by freezing and thawing, cryosurgery, infrared radiators and microwave methods. An excellent appraisal of these and numerous other thermal technologies in medicine has been provided by Diller et al. [14]. In parallel with clinical and laboratory-based thermal studies, mathematical and computational modelling of heat transfer in biosystems has emerged as a critical modern tool [15]. Simulation provides an inexpensive and powerful methodology for robust analysis of the behavior of complex organs and physiological processes involving not just heat transfer, but also mass transfer, sophisticated geometrical features and multi-physical fluid dynamics.

The electrically-conducting nature of blood and plasma (among other physiological liquids) has been known for many decades. The presence of iron in the haemoglobin molecule and also ions in streaming blood, chyme, synovial fluids etc, result in the response of such fluent media to externally imposed magnetic fields. The science of this phenomenon is known as magnetohydrodynamics. Togawa et al. [17] performed a seminal study confirming the electromotive force generated in unsteady hemodynamics with an externally imposed magnetic field, which contributed to the modern technique of magnetic extra-corporeal blood flow control. Further studies of clinical magnetohydrodynamic physiological transport include Saygih et al. [18] (hydromagnetic retention systems in prosthodontics in prostheses on buccal mucosal blood flow, Nijm et al. [19] (MRI procedures involving magnetohydrodynamic blood flow potential monitoring) and Valbona et al. [20] (magnetic pain therapy). Computational studies of magnetic biofluid dynamics include Mustapha et al. [21] (time-dependent multi-stenosed arterial flow with a marker and cell (MAC) code), Tripathi and Bég [22] (unsteady peristaltic thermal hydromagnetic pumping in intestinal dynamics using Mathematica software), Kenjereš and Righolt [24] (magnetic 
capturing of drug carriers in neuro-vascular transport), Rashidi et al. [24] (haemotological magnetic filtration simulation with a differential transform method) and Tripathi et al. [25] (electro-kinetic and magnetohydrodynamic peristaltic microfluidic device simulation).

A key feature of many biological liquids which is intrinsic to their proper functionality is rheology. Biorheology considers the non-Newtonian characteristics of biological liquids and how they contribute to complex roles in internal physiology and also external (natural) processes. Many sophisticated phenomena are involved in biorheology including viscoelasticity, viscoplasticity, memory effects, relaxation, retardation, spurt, normal stress differences etc. These cannot be simulated by the classical Newtonian model and modifications have to be made to the NavierStokes (viscous) flow equations. Coined by Copley [26], biorheology features in an astonishing range of systems including mucus deposition in coughing [27, 28], hemolysis [29], embryological propulsion [30], synovial lubrication and rheumatoid diseases [31, 32], and botanical protoplasmic streaming in plant cells such as Nitella and Chara [33].

A complex feature observed on biological surfaces is the cilium. Cilia constitute small but intricate appendage structures which protrude from vessel walls. Cilia [34] which average 10 microns in length can flex easily and assist therefore in many sophisticated biophysical transport mechanisms. They usually occur in high density arrays unlike flagella which usually appear in nature as single structures or pairs. Cilia beating mechanisms (metachronal) which control the direction of induced thrust, therefore differ significantly from flagellar beating. They exhibit whip-like- motions and appear on cells, plants, physiological organs, marine organisms. They exert a substantial role across the biological spectrum and feature in for example embryonic mechano-transduction processes, tracheal aerodynamics, coral reef systems, ventricular cerebrospinal fluid dynamics etc. Excellent studies of cilia dynamics have been presented by Lindemann and Leish [35] and Lindemann [36].

The beating mechanisms of cilia tend to be dominated by metachronal waves (these also dictate oscillations of flagella). Generally, the approaches for simulating ciliated flows fall into two broad categories. The first has become known as the envelope model (followed in the current article) and the second is the sub-layer model. In the envelope model the cilia are densely-packed, and the engulfing fluid medium interacts with a waving material surface enveloping the top of the layer. Further sub-categories of the envelop approach feature models of an actively driven semi-flexible filament (this aims to recreate actual cilia beat mechanisms) and beat shapes which are optimized 
based on pumping efficiency. In recent years rheological hydrodynamics with cilia effects have stimulated some attention. Interesting studies in this regard include Siddiqui et al. [37] (who employed the Ostwald-de Waele power law model), Maqbool et al. [38] (who considered the fractional Burgers model for ciliated propulsion in a tilted vessel) and Siddiqui et al. [39] (who simulated the epididymal fluid through the ductus efferentes of the male reproductive track by considering the Carreau fluid model in metachronal wave of cilia motion in an asymmetric tube). Several researchers have also considered combined heat transfer and fluid flow in ciliated propulsion with magnetohydrodynamic and other effects. Akbar et al. [40] studied the hydromagnetic visco-plastic metachronal propulsion with multiple slip effects. Shaheen and Nadeem [41] investigated viscoelastic Jeffrey six-constant nanofluid ciliated flow in an annular conduit. Abo-Elkhair et al. [42] investigated peristaltic cilia-assisted hydromagnetic pumping in a porous medium-filled channel.

In the present study, we examine for the first time, the magnetohydrodynamic pumping of a Jeffrey viscoelastic biofluid with convective heat transfer through a ciliated channel containing a homogenous, isotropic porous medium. Viscous dissipation effects are included. Darcy's law is employed to simulate porous medium drag effects. The two-dimensional momentum conservation partial differential equation and energy conservation equation are normalized with appropriate variables and transformed from a stationary coordinate system (laboratory or fixed frame) to a moving one (wave frame). The momentum equation is non-linear. A stream function is further used to reduce the boundary value problem. The emerging equations under appropriate transformations are solved by the semi-numerical/analytical Adomian decomposition method (ADM). The ADM solutions for the key flow characteristics i.e. velocity, temperature, pressure gradient and pressure rise are numerically evaluated in a symbolic software (MATHEMATICA). Streamline distributions are also presented. The influence of Hartmann number (magnetic parameter), Jeffrey first and second viscoelastic parameters, permeability parameter (modified Darcy number), and Brinkman number (viscous heating parameter) on velocity, temperature, pressure gradient and bolus dynamics is depicted via graphs. The simulations are relevant to the improved design of magnetic microfluidic fertilization devices [43] and also find applications in gastro-intestinal magnetic therapy [44]. 


\section{MATHEMATICAL FORMULATION}

The regime under investigation, as visualized in Fig. 1, examines the forced convective magnetohydrodynamic (MHD) flow of an incompressible viscoelastic physiological liquid through a ciliated channel of finite length, $L$. The inner walls of the channel are ciliated, and the channel contains a high-permeability porous medium (representative of deposits, debris etc in biomedical vessels). A constant strength of magnetic field, $B_{0}$, is applied transverse to the longitudinal axis of the channel. The collective motion of the cilia generates a metachronal wave in the axial direction of channel having wave speed, $c$, and wavelength, $\lambda$. The biofluid rheology is simulated with the robust Jeffery elastic-viscous model [45] for which the constitutive equation is:

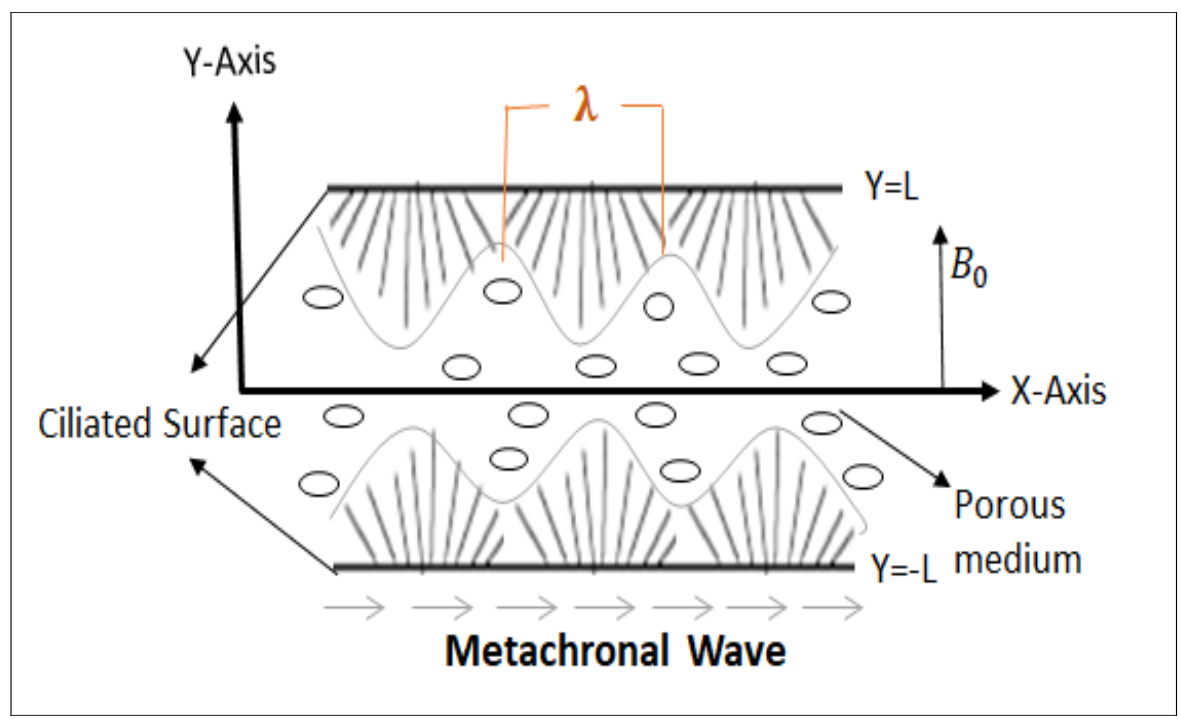

Fig. 1: Geometry of Problem

$$
\mathbf{T}=-p I+\mathbf{S}
$$

Where:

$$
\mathbf{S}=\frac{\mu}{1+\lambda_{1}}\left(\dot{\gamma}+\lambda_{2} \ddot{\gamma}\right)
$$

The momentum and energy equations in the fixed frame are given by: 
$\frac{\partial U}{\partial X}+\frac{\partial V}{\partial Y}=0$

$\rho\left(U \frac{\partial U}{\partial X}+V \frac{\partial U}{\partial Y}\right)=-\frac{\partial P}{\partial X}+\frac{\partial S_{X X}}{\partial X}+\frac{\partial S_{X Y}}{\partial Y}+(J \times B)_{X}+R_{X}$

$\rho\left(U \frac{\partial V}{\partial X}+V \frac{\partial V}{\partial Y}\right)=-\frac{\partial P}{\partial Y}+\frac{\partial S_{X Y}}{\partial X}+\frac{\partial S_{Y Y}}{\partial Y}+(J \times B)_{Y}+R_{Y}$

$$
\rho c_{p}\left(U \frac{\partial T}{\partial X}+\bar{V} \frac{\partial T}{\partial Y}\right)=S_{X X} \frac{\partial U}{\partial X}+S_{X Y}\left(\frac{\partial U}{\partial Y}+\frac{\partial V}{\partial X}\right)+S_{Y Y} \frac{\partial V}{\partial Y}
$$

$$
k\left(\frac{\partial^{2} T}{\partial X^{2}}+\frac{\partial^{2} T}{\partial Y^{2}}\right)
$$

Here:

$$
\begin{aligned}
& S_{X X}=\frac{2 \mu}{1+\lambda_{1}}\left[1+\lambda_{2}\left(U \frac{\partial}{\partial X}+V \frac{\partial}{\partial Y}\right)\right] \frac{\partial U}{\partial X} \\
& S_{X Y}=\frac{\mu}{1+\lambda_{1}}\left[1+\lambda_{2}\left(U \frac{\partial}{\partial X}+V \frac{\partial}{\partial Y}\right)\right]\left(\frac{\partial U}{\partial Y}+\frac{\partial V}{\partial X}\right), \\
& S_{Y Y}=\frac{2 \mu}{1+\lambda_{1}}\left[1+\lambda_{2}\left(U \frac{\partial}{\partial X}+V \frac{\partial}{\partial Y}\right)\right] \frac{\partial V}{\partial X} \\
& (J \times B)_{X}=-\sigma B_{0}^{2}, \quad(J \times B)_{Y}=0
\end{aligned}
$$




$$
R_{X}=-\frac{\mu \phi}{k} U, \quad R_{Y}=-\frac{\mu \phi}{k} V
$$

The envelope of the cilia tips can be written as:

$$
\begin{aligned}
& Y=H(X, t)= \pm\left[a+\varepsilon a \cos \left(\frac{2 \pi}{\lambda}\right)(X-c t)\right]= \pm L \\
& X=F(X, t)=X_{0}+\varepsilon a \alpha \sin \left(\frac{2 \pi}{\lambda}\right)(X-c t),
\end{aligned}
$$

The transformation from fixed to wave frame are given by

$$
\begin{gathered}
\bar{x}=X-c t, \quad \bar{y}=Y, \quad \bar{u}=U-c, \quad \bar{v}=V, \\
\bar{p}(\bar{x}, \bar{y}, \bar{t})=P(X, Y, t),
\end{gathered}
$$

The following non-dimensional variables are implemented:

$$
\begin{aligned}
& x=\frac{\bar{x}}{\lambda}, \quad y=\frac{\bar{y}}{a}, \quad u=\frac{\bar{u}}{c}, \quad v=\frac{\lambda \bar{v}}{a c}, \\
& p=\frac{a^{2} \bar{p}}{c \lambda \mu}, \quad t=\frac{c \bar{t}}{\lambda}, \quad h=\frac{L}{a}, \quad \operatorname{Re}=\frac{\rho c a}{\mu}, \\
& \beta=\frac{a}{\lambda}, \quad M^{2}=\frac{\sigma B_{0}^{2} a^{2}}{\mu}, \quad \frac{1}{K}=\frac{\phi a^{2}}{k} .
\end{aligned}
$$

Here all parameters are defined in the nomenclature. With the help of Eqns. (7)-(15) and employing the low Reynolds number approximation from lubrication theory, Eqns. (3)-(6) take the following form: 


$$
\begin{aligned}
\frac{\partial u}{\partial x}+ & \frac{\partial v}{\partial y}=0 \\
\frac{\partial p}{\partial x}= & \frac{1}{1+\lambda_{1}} \frac{\partial}{\partial y}\left(1+\frac{\lambda_{2} \beta c}{a}\left(u \frac{\partial}{\partial x}+v \frac{\partial}{\partial y}\right)\right) \frac{\partial u}{\partial y} \\
& -\left(M^{2}+\frac{1}{K}\right)(u+1) \\
\frac{\partial p}{\partial y}= & 0 \\
\frac{\partial^{2} \theta}{\partial y^{2}}= & -\frac{B r}{1+\lambda_{1}}\left(1+\frac{\lambda_{2} \beta c}{a}\left(u \frac{\partial}{\partial x}+v \frac{\partial}{\partial y}\right)\left(\frac{\partial u}{\partial y}\right)^{2}\right)
\end{aligned}
$$

The associated boundary conditions emerge as:

$$
\begin{aligned}
& u(h)=-1-2 \pi \varepsilon \alpha \beta \cos 2 \pi x \\
& v(h)=2 \pi \varepsilon \sin 2 \pi x+\beta(2 \pi \varepsilon)^{2} \alpha \sin 2 \pi x \cos 2 \pi x, \\
& \theta(h)=0, \quad \text { at } y=h \\
& \frac{\partial u}{\partial y}=0, \frac{\partial \theta}{\partial y}=0 \text { at } \quad y=0 .
\end{aligned}
$$

Here $h=1+\varepsilon \operatorname{Cos} 2 \pi x$. The stream function $\psi$ is defined as:

$$
u=\frac{\partial \psi}{\partial y}, \quad v=-\frac{\partial \psi}{\partial x}
$$

Eqns. (16)-(19) and boundary conditions (20) in terms of $\psi$ take the following form: 


$$
\begin{aligned}
\frac{\partial^{4} \psi}{\partial y^{4}}= & -\frac{\lambda_{2} \beta c}{a} \frac{\partial^{2}}{\partial y^{2}}\left(\frac{\partial \psi}{\partial y} \frac{\partial}{\partial x}-\frac{\partial \psi}{\partial x} \frac{\partial}{\partial y}\right) \frac{\partial^{2} \psi}{\partial y^{2}} \\
& +\left(M^{2}+\frac{1}{K}\right)\left(1+\lambda_{1}\right) \frac{\partial^{2} \psi}{\partial y^{2}} \\
\frac{\partial^{2} \theta}{\partial y^{2}}= & -\frac{B r}{1+\lambda_{1}}\left(1+\frac{\lambda_{2} \beta c}{a}\left(\frac{\partial \psi}{\partial y} \frac{\partial}{\partial x}-\frac{\partial \psi}{\partial x} \frac{\partial}{\partial y}\right)\left(\frac{\partial^{2} \psi}{\partial y^{2}}\right)^{2}\right),
\end{aligned}
$$

The boundary conditions can be expressed as:

$$
\begin{aligned}
\psi & =0, \quad \frac{\partial^{2} \psi}{\partial y^{2}}=0, \quad \frac{\partial \psi}{\partial x}=0, \quad \frac{\partial \theta}{\partial y}=0, \quad \text { at } \quad y=0, \\
\psi & =q, \quad \frac{\partial \psi}{\partial y}=-1-2 \pi \varepsilon \alpha \beta \cos 2 \pi x, \quad \theta=0, \\
\frac{\partial \psi}{\partial x} & =2 \pi \varepsilon \sin 2 \pi x+\beta(2 \pi \varepsilon)^{2} \alpha \sin 2 \pi x \cos 2 \pi x, \quad \text { at } \quad y=h .
\end{aligned}
$$

Here $q$ is flux and $Q$, the volumetric flow rate, is related to the flux by the following relation:

$$
Q=\int_{0}^{h}\left(\frac{\partial \psi}{\partial y}+1\right) d y=q+h
$$

The time mean volumetric flow rate $\bar{Q}$ in a fixed frame is defined as:

$$
\bar{Q}=\frac{1}{T} \int_{0}^{h}(q+h) d \bar{t}=\int_{0}^{1}(q+h) d t=q+1
$$

\section{ADM SOLUTION}

Many advanced computational and semi-computational methods may be employed to solve the 
transformed boundary value problem defined by Eqns. (22) and (23) under boundary conditions (24). These include homotopy methods, spectral methods, variational iterative methods and finite element methods. We elect to employ the Adomian decomposition method (ADM). Introduced by American mathematician, Adomian [46] uses very precise polynomial expansions to achieve faster convergence than many other procedures. An advantage of ADM is that it can provide analytical approximations or an approximated solution to a wide class of nonlinear equations without linearization, perturbation closure approximations or discretization methods. ADM deploys an infinite series solution for the unknown functions and utilizes recursive relations. Recent applications of this technique in complex biological flow problems include Bég et al. [47] (for smart lubrication squeeze films), Bég [48] (swirling nanofluid bioreactors), Bég [49] (rotating bio-nano-polymer coating flows) and Schit et al. [50] (hydromagnetic peristaltic blood flow). Rewriting Eqns. (22) and (23) in terms of Adomian operators we have:

$$
\begin{aligned}
\psi= & \varphi_{0}+L_{1}^{-1}\left(-\lambda_{2} \beta \frac{\partial^{2}}{\partial y^{2}}\left(\frac{\partial \psi}{\partial y} \frac{\partial}{\partial x}-\frac{\partial \psi}{\partial x} \frac{\partial}{\partial y}\right) \frac{\partial^{2} \psi}{\partial y^{2}}\right) \\
& +L_{1}^{-1}\left(\left(M^{2}+\frac{1}{K}\right)\left(1+\lambda_{1}\right) \frac{\partial^{2} \psi}{\partial y^{2}}\right), \\
\theta= & \varphi_{1}-\frac{B r}{1+\lambda_{1}} L_{2}^{-1}\left(\left(1+\lambda_{2} \beta\left(\frac{\partial \psi}{\partial y} \frac{\partial}{\partial x}-\frac{\partial \psi}{\partial x} \frac{\partial}{\partial y}\right)\left(\frac{\partial^{2} \psi}{\partial y^{2}}\right)^{2}\right)\right)
\end{aligned}
$$

Here the inverse operators are defined as:

$$
\begin{aligned}
& L_{1}^{-1}(.)=\iiint \int(.) d y, \\
& L_{2}^{-1}(.)=\iint(.) d y,
\end{aligned}
$$


The linear term $\psi(x, y)$ is decomposed in term of an infinite series of components through the following expression:

$$
\psi(x, y)=\sum_{n=0}^{\infty} \psi_{n}(x, y)
$$

The nonlinear term $N \psi(x, y)$ can be decomposed into Adomian polynomials and satisfies:

$$
A_{n}=1 \frac{1}{n !} \frac{d^{n}}{d \lambda^{m}}\left[N\left(\sum_{i=0}^{\infty} \lambda_{i} \psi_{i}\right)\right]_{\lambda=0}, \quad n=0,1,2,3, \ldots,
$$

This leads to:

$$
\begin{aligned}
& A_{0}=-\frac{\partial \psi_{0}}{\partial x} \frac{\partial^{5} \psi_{0}}{\partial y^{5}}+\frac{\partial \psi_{0}}{\partial y} \frac{\partial^{5} \psi_{0}}{\partial x \partial y^{4}}+2 \frac{\partial^{2} \psi_{0}}{\partial y^{2}} \frac{\partial^{4} \psi_{0}}{\partial x \partial y^{3}} \\
& -2 \frac{\partial^{4} \psi_{0}}{\partial y^{4}} \frac{\partial^{2} \psi_{0}}{\partial x \partial y} \\
& A_{1}=-\frac{\partial \psi_{0}}{\partial x} \frac{\partial^{5} \psi_{1}}{\partial y^{5}}-\frac{\partial \psi_{1}}{\partial x} \frac{\partial^{5} \psi_{0}}{\partial y^{5}}+\frac{\partial \psi_{0}}{\partial y} \frac{\partial^{5} \psi_{1}}{\partial x \partial y^{4}} \\
& +\frac{\partial \psi_{1}}{\partial y} \frac{\partial^{5} \psi_{0}}{\partial x \partial y^{4}}+2 \frac{\partial^{2} \psi_{0}}{\partial y^{2}} \frac{\partial^{4} \psi_{1}}{\partial x \partial y^{3}}+2 \frac{\partial^{2} \psi_{1}}{\partial y^{2}} \frac{\partial^{4} \psi_{0}}{\partial x \partial y^{3}} \\
& -2 \frac{\partial^{4} \psi_{0}}{\partial y^{4}} \frac{\partial^{2} \psi_{1}}{\partial x \partial y}-2 \frac{\partial^{4} \psi_{1}}{\partial y^{4}} \frac{\partial^{2} \psi_{0}}{\partial x \partial y} \\
& A_{2}=-\frac{\partial \psi_{0}}{\partial x} \frac{\partial^{5} \psi_{2}}{\partial y^{5}}-\frac{\partial \psi_{1}}{\partial x} \frac{\partial^{5} \psi_{1}}{\partial y^{5}}-\frac{\partial \psi_{2}}{\partial x} \frac{\partial^{5} \psi_{0}}{\partial y^{5}} \\
& +\frac{\partial \psi_{0}}{\partial y} \frac{\partial^{5} \psi_{2}}{\partial x \partial y^{4}}+\frac{\partial \psi_{1}}{\partial y} \frac{\partial^{5} \psi_{1}}{\partial x \partial y^{4}}+\frac{\partial \psi_{2}}{\partial y} \frac{\partial^{5} \psi_{0}}{\partial x \partial y^{4}} \\
& +2 \frac{\partial^{2} \psi_{0}}{\partial y^{2}} \frac{\partial^{4} \psi_{2}}{\partial x \partial y^{3}}+2 \frac{\partial^{2} \psi_{1}}{\partial y^{2}} \frac{\partial^{4} \psi_{1}}{\partial x \partial y^{3}}+2 \frac{\partial^{2} \psi_{2}}{\partial y^{2}} \frac{\partial^{4} \psi_{0}}{\partial x \partial y^{3}} \\
& -2 \frac{\partial^{4} \psi_{0}}{\partial y^{4}} \frac{\partial^{2} \psi_{2}}{\partial x \partial y}-2 \frac{\partial^{4} \psi_{1}}{\partial y^{4}} \frac{\partial^{2} \psi_{1}}{\partial x \partial y}-2 \frac{\partial^{4} \psi_{2}}{\partial y^{4}} \frac{\partial^{2} \psi_{0}}{\partial x \partial y}
\end{aligned}
$$




$$
\begin{gathered}
\varphi_{0}=c_{1}+c_{2} y+c_{3} \frac{y^{2}}{2 !}+c_{4} \frac{y^{3}}{3 !}, \\
\varphi_{1}=d_{1}+d_{2} y
\end{gathered}
$$

Here $c_{1}, c_{2}, c_{3}, c_{4}, d_{1}, d_{2}$ are constants of integration and can be found by using the boundary conditions given in Eqn. (24). Now by decomposing the linear and the non-linear terms in the form of infinite series, we get:

$$
\begin{aligned}
\sum_{n=0}^{\infty} \psi_{n}= & \varphi_{0, n}+L_{1}^{-1} \sum_{n=0}^{\infty}\left(-\lambda_{2} \beta \frac{\partial^{2}}{\partial y^{2}}\left(\frac{\partial \psi_{n}}{\partial y} \frac{\partial}{\partial x}-\frac{\partial \psi_{n}}{\partial x} \frac{\partial}{\partial y}\right) \frac{\partial^{2} \psi_{n}}{\partial y^{2}}\right) \\
& +L_{1}^{-1} \sum_{n=0}^{\infty}\left(\left(M^{2}+\frac{1}{K}\right)\left(1+\lambda_{1}\right) \frac{\partial^{2} \psi_{n}}{\partial y^{2}}\right), \\
\sum_{n=0}^{\infty} \theta_{n}= & \varphi_{1, n}-\frac{B r}{1+\lambda_{1}} L_{2}^{-1}\left(\left(1+\lambda_{2} \beta\left(\begin{array}{c}
\frac{\partial \psi_{n}}{\partial y} \frac{\partial}{\partial x} \\
-\frac{\partial \psi_{n}}{\partial x} \frac{\partial}{\partial y}
\end{array}\right)\left(\frac{\partial^{2} \psi_{n}}{\partial y^{2}}\right)^{2}\right)\right)
\end{aligned}
$$

Following ADM, we obtain:

$$
\begin{aligned}
\psi_{0}= & \left(\frac{3 q-h u(h)}{2 h}\right) y-\left(\frac{q-h u(h)}{2 h^{3}}\right) y^{3} \\
\theta_{0}= & \frac{1}{10 h^{4}\left(1+\lambda_{1}\right)}\left[B r \left(27 \beta q^{3} \lambda_{2} h^{\prime}+h\left(-42 \beta q^{2} \lambda_{2} u(h) h^{\prime}\right.\right.\right. \\
& +h\left(-5 a h^{4}+18 \beta q \lambda_{2} u^{2}(h) h^{\prime}-3 \beta h \lambda_{2} u^{3}(h) h^{\prime}\right. \\
& \left.\left.\left.+3 \beta \lambda_{2}(q-h u(h))(3 q+2 h u(h)) u^{\prime}(h)\right)\right)\right]+\frac{B r}{2+2 \lambda_{1}} y^{2} \\
& -\frac{1}{2 h^{8}\left(1+\lambda_{1}\right)}\left[\begin{array}{c}
3 B r \beta \lambda_{2}(q-h u(h))\left(3 q^{2}-3 h q u(h)\right. \\
\left.+h^{2} u^{2}(h) h^{\prime}+h^{2} q u^{\prime}(h)\right)
\end{array}\right] y^{4} \\
& +\frac{1}{5 h^{10}\left(1+\lambda_{1}\right)}\left[\begin{array}{c}
3 B r \beta \lambda_{2}(q-h u(h))^{2} \\
\times\left((3 q-2 h u(h)) h^{\prime}+h^{2} u^{\prime}\right)
\end{array}\right] y^{6},
\end{aligned}
$$




$$
\begin{aligned}
\psi_{n}= & L_{1}^{-1}\left(-\lambda_{2} \beta \frac{\partial^{2}}{\partial y^{2}}\left(\frac{\partial \psi_{n-1}}{\partial y} \frac{\partial}{\partial x}-\frac{\partial \psi}{\partial x} \frac{\partial}{\partial y}\right) \frac{\partial^{2} \psi_{n-1}}{\partial y^{2}}\right) \\
& +L_{1}^{-1}\left(\left(M^{2}+\frac{1}{K}\right)\left(1+\lambda_{1}\right) \frac{\partial^{2} \psi_{n-1}}{\partial y^{2}}\right), \quad n \geq 1 \\
\theta_{n}= & -\frac{B r}{1+\lambda_{1}} L_{2}^{-1}\left(\left(1+\lambda_{2} \beta\left(\begin{array}{c}
\frac{\partial \psi_{n}}{\partial y} \frac{\partial}{\partial x} \\
-\frac{\partial \psi_{n}}{\partial x} \frac{\partial}{\partial y}
\end{array}\right)\left(\frac{\partial^{2} \psi_{n}}{\partial y^{2}}\right)^{2}\right)\right)
\end{aligned}
$$

The solution in the form of $\psi$ can be written as:

$$
\begin{aligned}
\psi= & \sum_{n=0}^{\infty} \psi_{n}=\psi_{0}+\psi_{1}+\psi_{2}+\psi_{3}+\ldots=A_{11}(x) y+A_{12}(x) y^{3} \\
& +A_{13}(x) y^{5}+A_{14}(x) y^{7}+A_{15}(x) y^{9}+\ldots,
\end{aligned}
$$

Using Eqn. (30) in Eqn. (22) the temperature solution may be determined:

$$
\begin{aligned}
\theta= & B_{1}(x)+B_{2}(x) y^{4}+B_{3}(x) y^{6}+B_{4}(x) y^{8}+B_{5}(x) y^{10} \\
& +B_{6}(x) y^{12}+B_{7}(x) y^{14}+B_{8}(x) y^{16}+B_{9}(x) y^{18} \\
& +B_{10}(x) y^{20}+B_{11}(x) y^{22}+B_{12}(x) y^{24}+\ldots,
\end{aligned}
$$

Here $A_{11}(x), A_{12}(x) \ldots B_{12}(x)$ are given in the Appendix. The pressure gradient $(\partial p / \partial x$ i.e. $d p / d x)$ by using Eqn. (44) and Eqn. (44) in Eqn. (17) as follows:

$$
\begin{aligned}
\frac{d p}{d x}= & \frac{1}{1+\lambda_{1}} \frac{\partial}{\partial y}\left(1+\lambda_{2} \beta\left(\frac{\partial \psi}{\partial y} \frac{\partial}{\partial x}-\frac{\partial \psi}{\partial x} \frac{\partial}{\partial y}\right)\right) \frac{\partial^{2} \psi}{\partial y^{2}} \\
& -\left(M^{2}+\frac{1}{K}\right)\left(\frac{\partial \psi}{\partial y}+1\right),
\end{aligned}
$$


The pressure rise per wavelength can be obtained by integrating Eqn. (46) as:

$$
\Delta p=\int_{0}^{1} \frac{d p}{d x} d x
$$

However, the numerical value of the integral is evaluated using the symbolic software MATHEMATICA.

\section{RESULTS AND DISCUSSION}

The influence of physical parameters of interest on axial velocity $u(x, y)$, axial pressure gradient $(d p / d x)$, pressure rise $(\Delta P)$, temperature profile, $\theta(x, y)$ and stream function $\psi(x, y)$ are displayed graphically in Figs. 2-9.

\section{Axial Velocity:}

In Figs.2(a-d) the effect of Hartmann number $(M)$, permeability parameter $(K)$, Jeffrey first and second viscoelastic parameters $\left(\lambda_{1}, \lambda_{2}\right)$ on the horizontal velocity $u(x, y)$. It is observed from this figure that behavior of velocity is not same in the centre and near to the wall of channel. Figs.2a and $2 \mathrm{c}$ show that the axial velocity decreases in the region $-0.38<y<0.38$ otherwise significant variation is not seen with the increase in $M$ and $\lambda_{1}$ whereas the converse behavior can be seen in Figs. $2 \mathrm{~b}$ and $2 \mathrm{~d}$ with an increase in $K$ and $\lambda_{2}$.

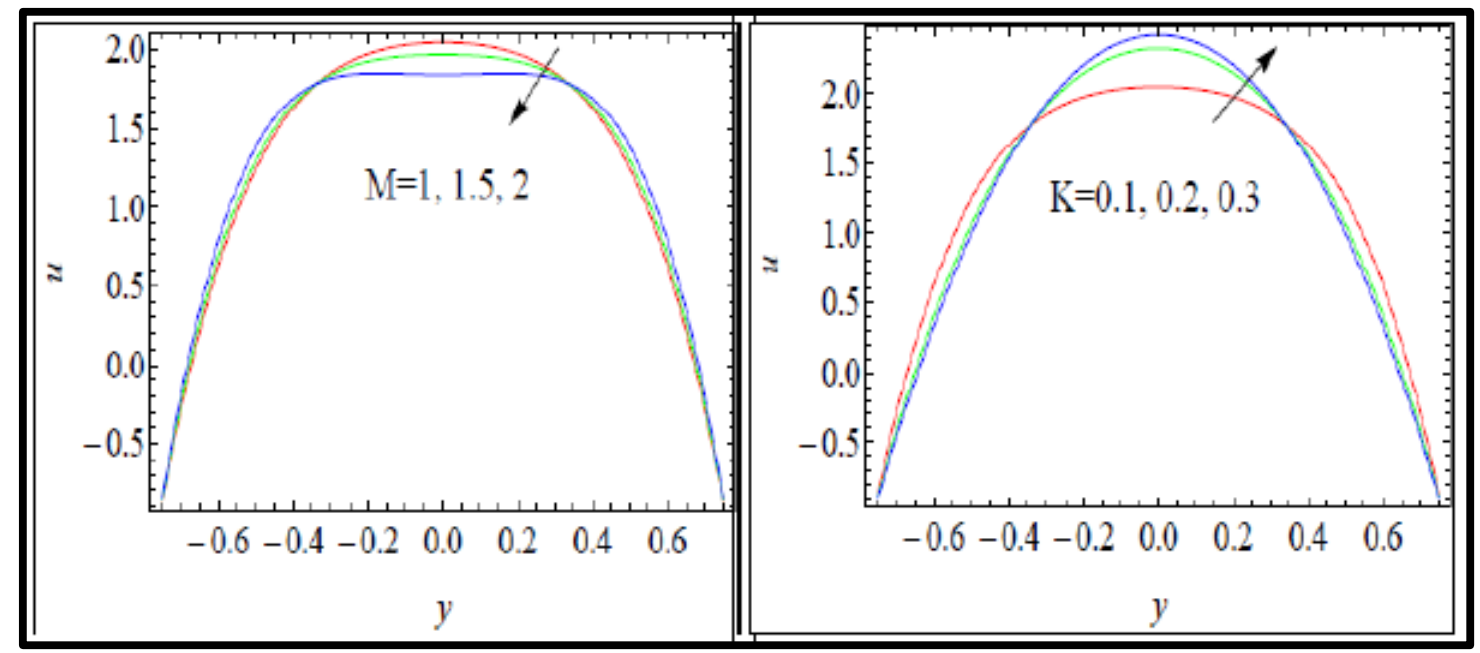




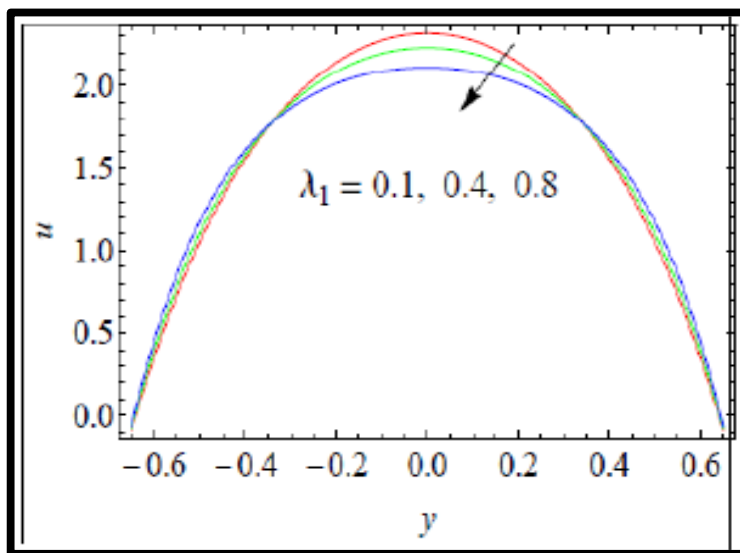

C

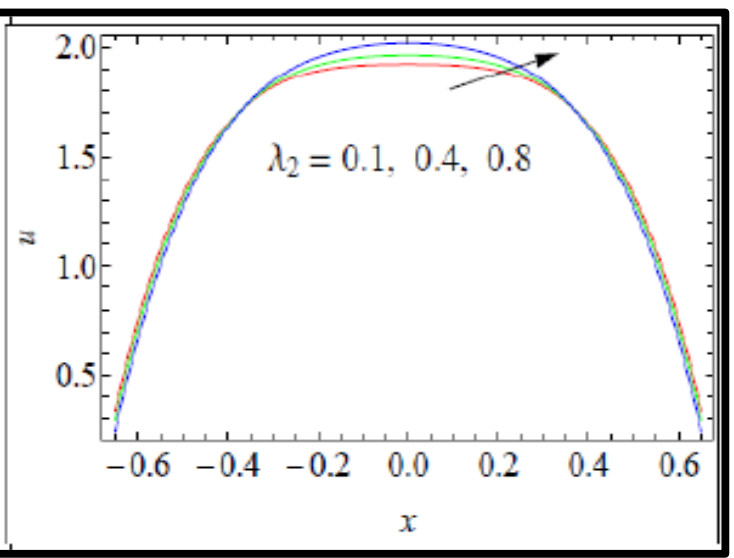

d

Fig.2(a-d): Influence of $M, K, \lambda_{1}, \lambda_{2}$ on axial velocity $u(x, y)$.

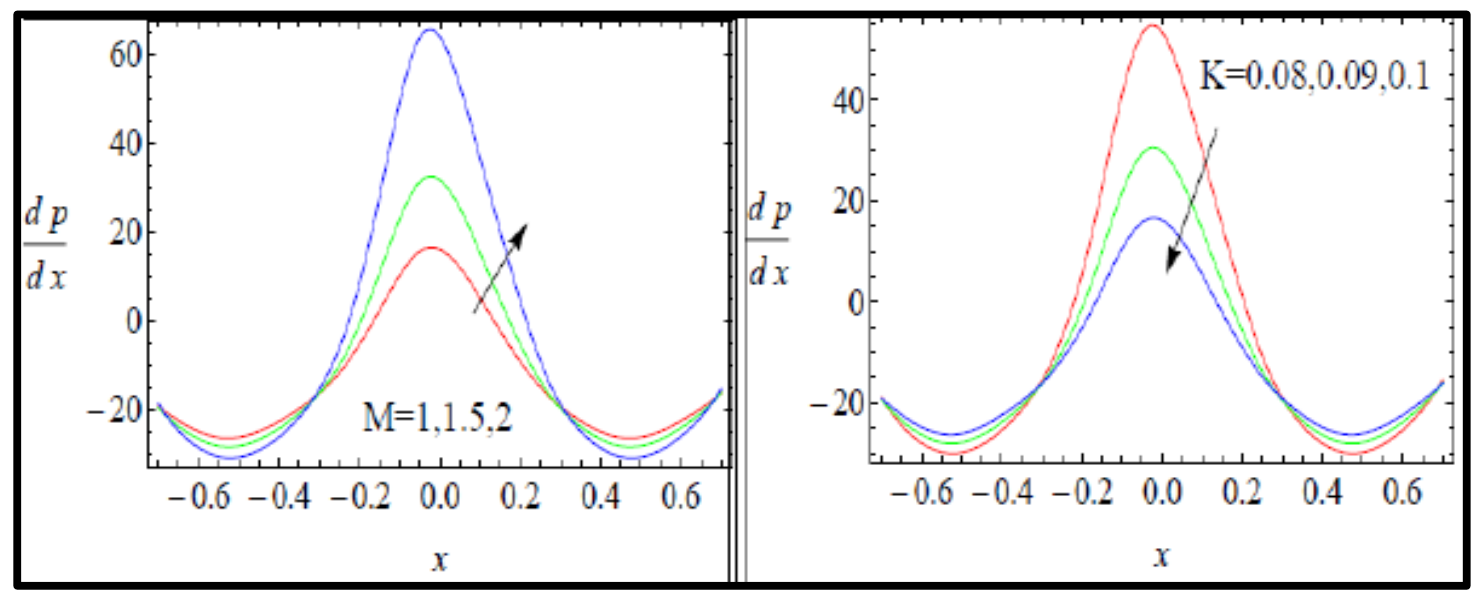

a

b

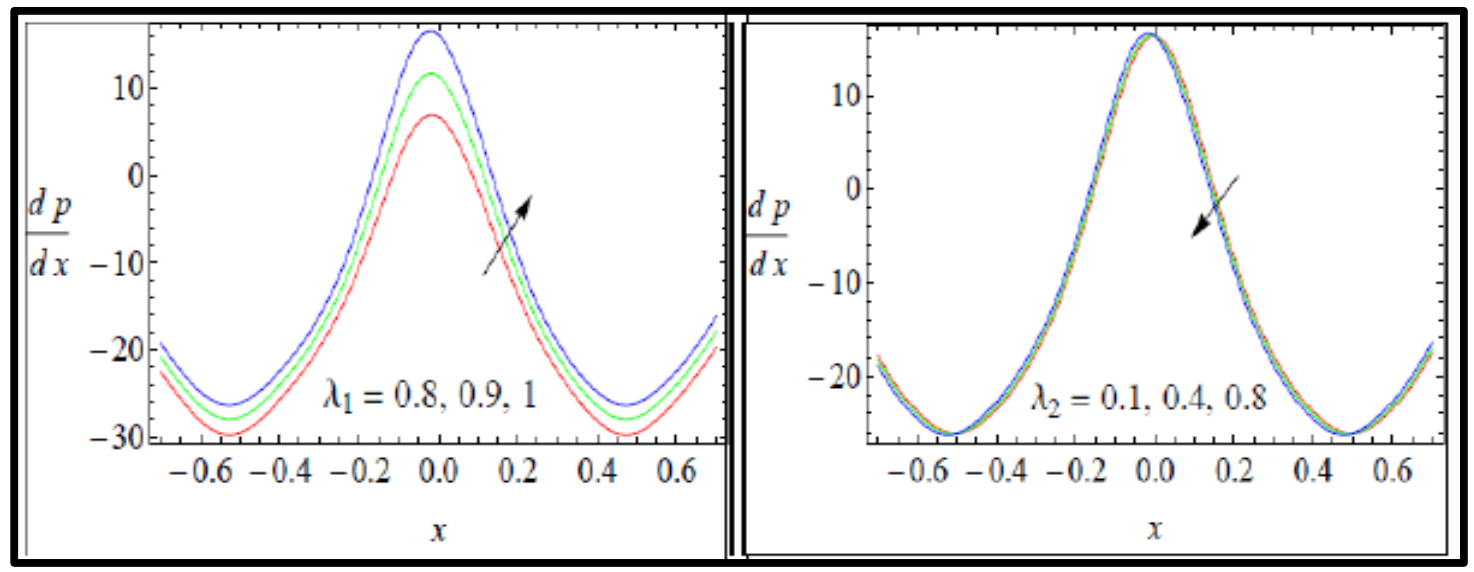

C

d

Fig.3(a-d): Influence of $M, K, \lambda_{1}, \lambda_{2}$ on axial pressure gradient ( $\left.d p / d x\right)$ 

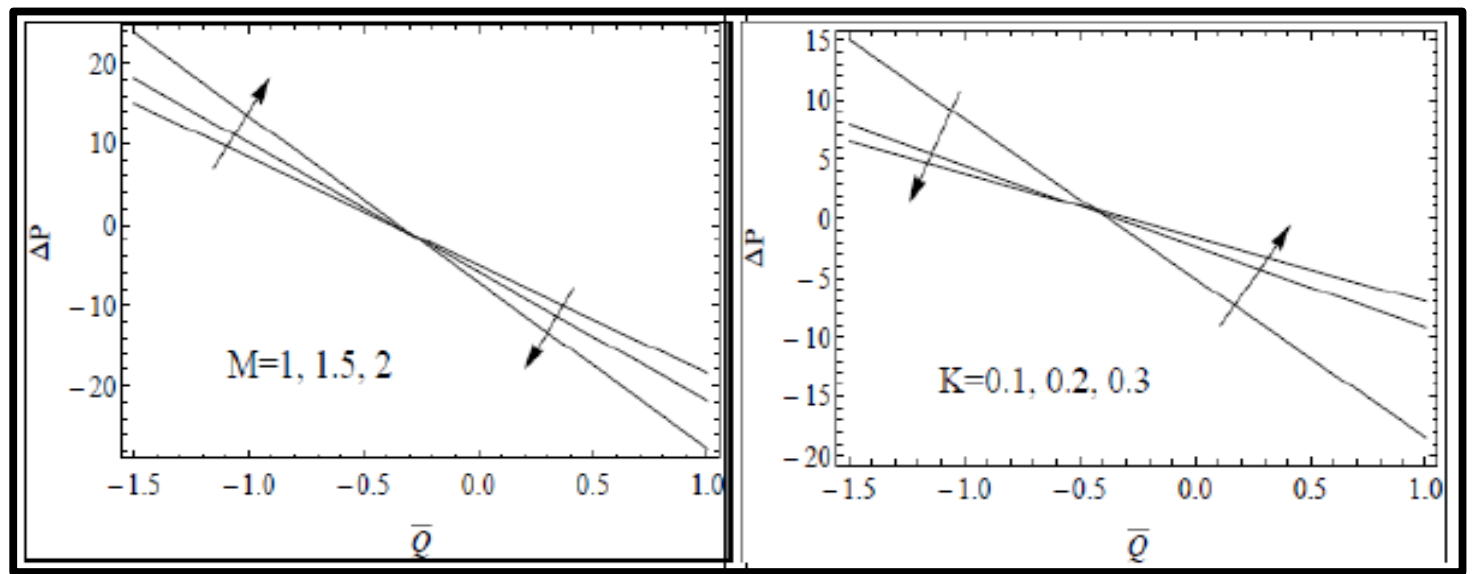

a

b

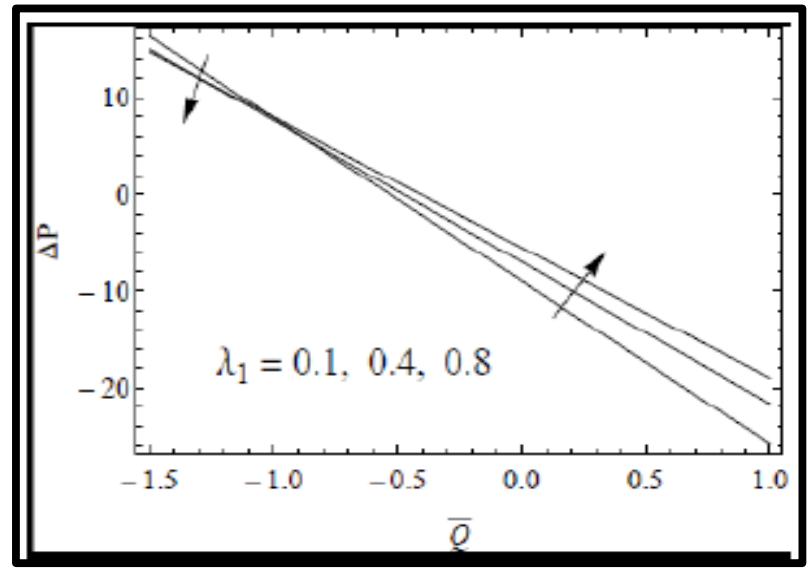

C

Fig.4(a-c): Influence of $M, K, \lambda_{1}, \lambda_{2}$ on pressure rise $(\Delta P)$ with time mean volumetric flow rate $\left(Q^{-}\right)$.
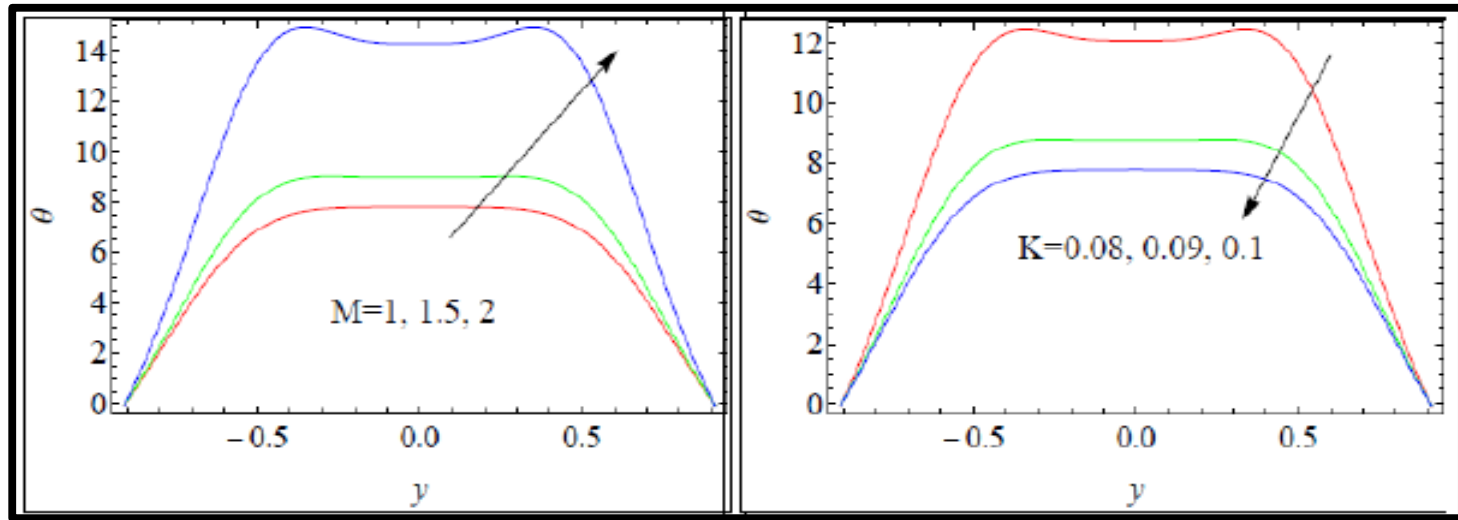

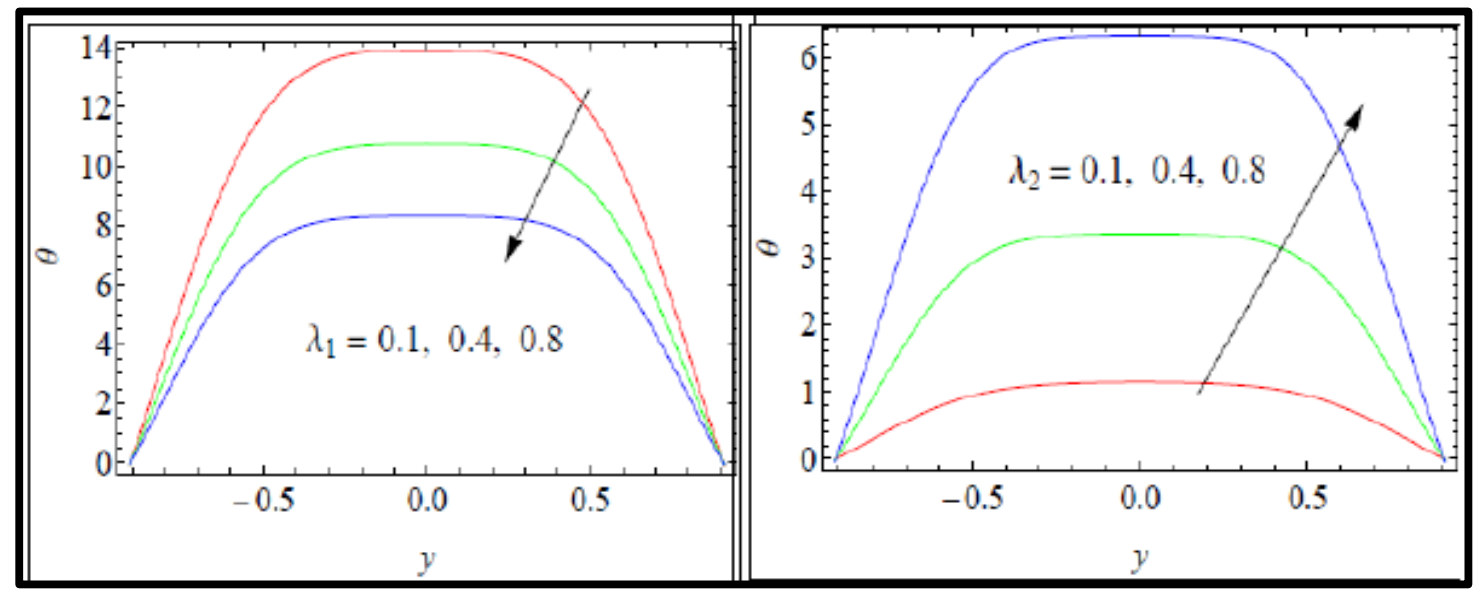

C

d

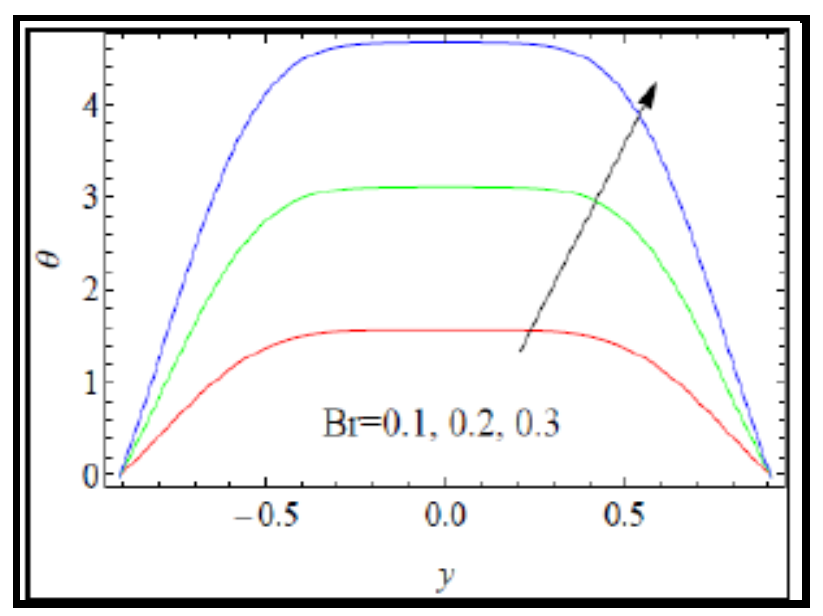

e

Fig.5(a-e): Influence $M, K, \lambda_{1}, \lambda_{2}, \operatorname{Br}$ on temperature profile $\theta(\mathrm{x} ; \mathrm{y})$.

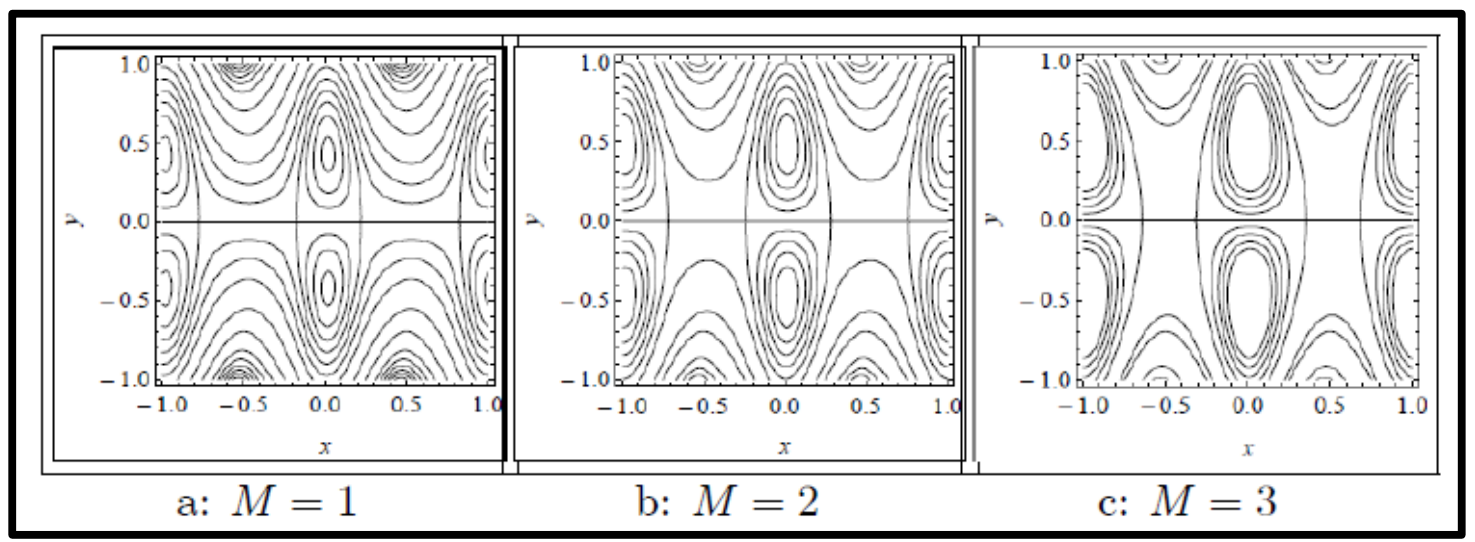

Fig.6(a-c): Influence of Hartmann number $(M)$ on stream function with $\alpha=0: 3 ; \beta=0: 1 ; \varepsilon=0: 3$; $Q=2 ; K=0: 1 ; \lambda_{1}=\lambda_{2} .=1$. 


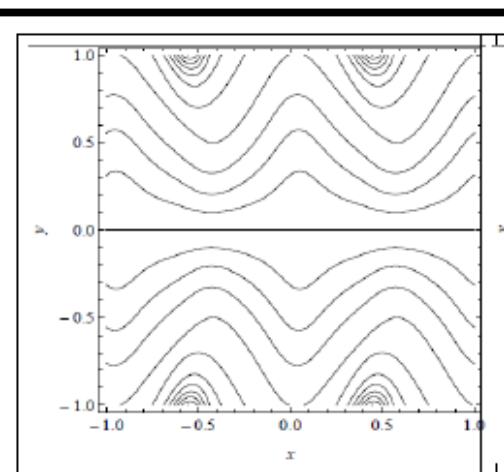

a: $K=0.2$



b: $K=0.3$

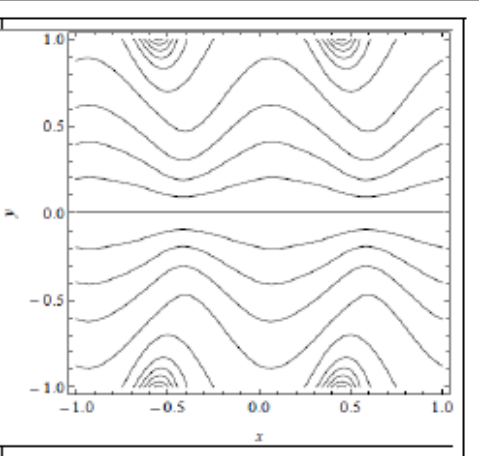

c: $K=0.4$

Fig.7(a-c): Influence of permeability parameter $(K)$ on stream function with $\alpha=0: 3 ; \beta=0: 1 ; \varepsilon$ $=0: 3 ; Q=2 ; M=1 ; \lambda_{1}=\lambda_{2}=1$.

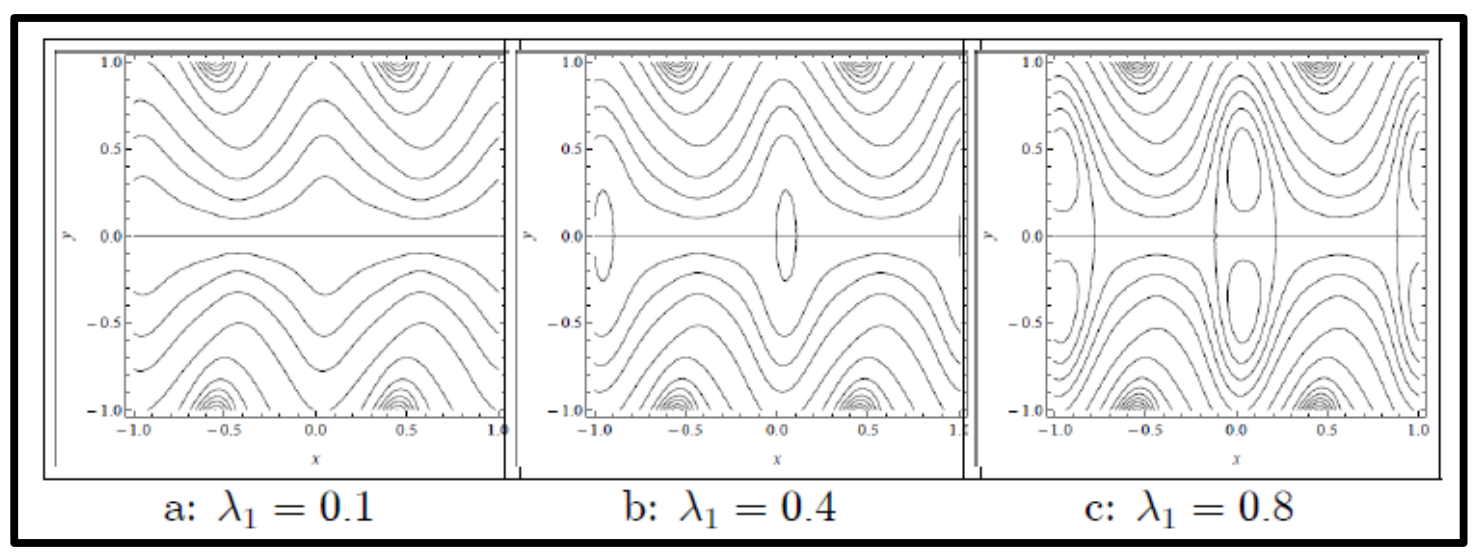

Fig.8(a-c): Influence of Jeffrey parameter $\lambda_{1}$ on stream function with $\alpha=0: 3 ; \beta=0: 1 ; \varepsilon=0: 3$; $Q=2 ; M=1 ; K=0.1, \lambda_{2}=1$.

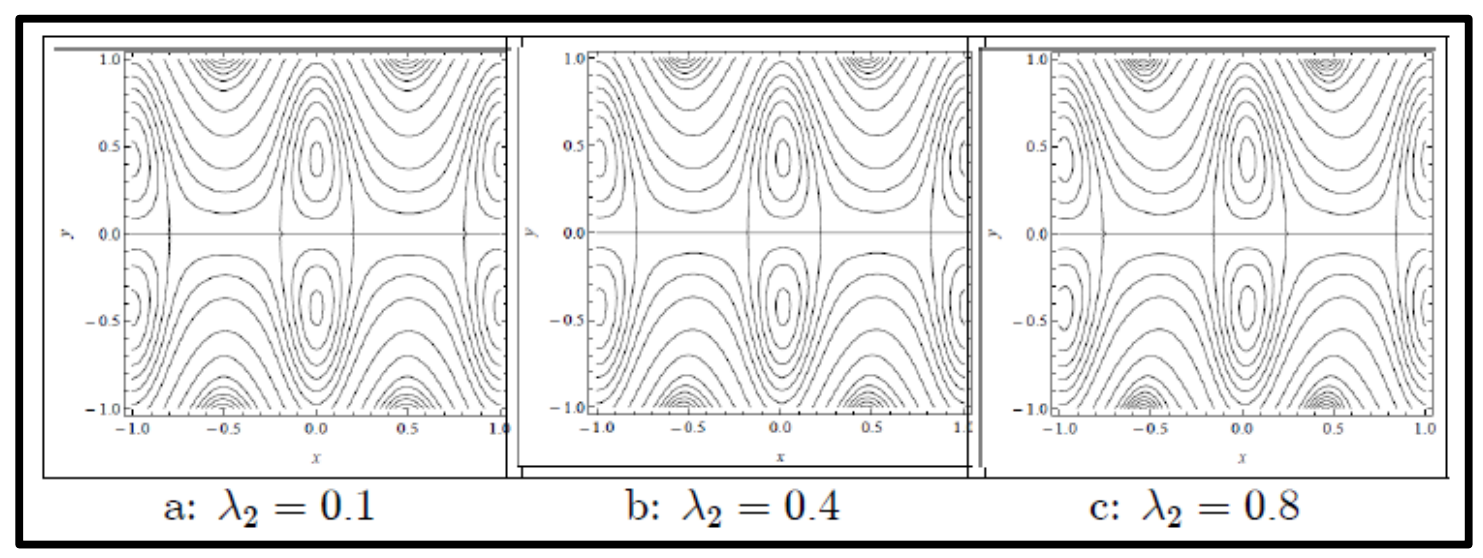

Fig.8(a-c): Influence of Jeffrey parameter $\lambda_{2}$ on stream function with $\alpha=0: 3 ; \beta=0: 1 ; \varepsilon=0: 3$; $Q=2 ; M=1 ; K=0.1, \lambda_{1}=1$. 


\section{Pumping characteristics:}

Fig.3 shows that the pressure gradient has a periodic nature and attains its maximum at centre of the channel from where it decreases rapidly as we progress from the core zone. Similar to velocity field the behavior of pressure gradient exhibits some variation throughout the region. It can be observed from Figs. 3a that pressure gradient is boosted at the centre and depressed near the walls

with increasing Hartmann number. The magnetic field effect is enhanced with greater Hartmann number and this generates deceleration in the flow. The inverse relation between velocity and pressure manifests in an elevation in pressure gradient in the core flow. With increasing permeability $(K)$, although velocity is enhanced (fig. 2b), the converse effect is induced in pressure gradient (Fig. 3c). The Darcy resistance term in Eqn. (22) is inversely proportional to permeability. Increasing $K$ values decrease the impedance to flow and result in an acceleration and a drop in the pressure gradient. Figs. $3 \mathrm{c}$ and d show that the first $\left(\lambda_{1}\right)$, and second Jeffery $\left(\lambda_{2}\right)$ parameters induce respectively an enhancement and suppression in the pressure gradient, although the first parameter has a much more profound effect. The first parameter denotes the ratio of the relaxation to retardation times of the bio-rheological fluid whereas the second parameter designates purely retardation time. When $\lambda_{1}=1$ both relaxation and retardation time are exactly equivalent. However, we have considered values less than unity which are more representative of physiologicalfluids [51]. When $\lambda_{1}<1$ the retardation time exceeds the relaxation time. This implies that the biofluid responds faster with the removal of stress and returns quicker to its unperturbed state. This influences pressure gradient (and velocity field)

Fig.4 shows the effect of different values of Hartmann number $(M)$, permeability parameter $(K)$, and the ratio of retardation to relaxation times $\left(\lambda_{1}\right)$ on pressure rise $(\Delta P)$ versus $\bar{Q}$. It is observed from these figures that there is a linear relation between pressure rise $\Delta P$ and volumetric flow rate $\bar{Q}$. Fig. 4a shows that pressure rise increases with Hartmann number, $M$, in the region $-1.5<Q<$ -0.3 and decreases in the range $-0.4<Q<1$ whereas, it decreases with greater permeability (lesser Darcian resistance) in the range $-1.5<Q<-0.4$. The effect of $\lambda_{1}$ on pressure rise is to decrease it in the region $-1.5<Q<-1.0$. However, the contrary effect is induced in the range $-1.5<Q<1.0$ with increasing first Jeffery viscoelastic parameter.

Temperature profile: 
Fig. 5(a-e) is plotted to analyze the influence of Hartmann number $(M)$, permeability parameter $(K)$, Brinkman number $(B r)$, ratio of relaxation to retardation times $\left(\lambda_{1}\right)$ and retardation time $\left(\lambda_{2}\right)$ on temperature distribution across the channel. The behavior of the temperature profile is similar to that of the velocity profile although the profiles are significantly more plateau-like in the core zone of the channel. Significantly less variation in profiles is observed near the channel walls. Increasing Hartmann number (fig. 5a) induces a strong elevation in temperatures across the channel. The supplementary work expended by the biofluid in dragging against the action of the applied magnetic field is dissipated as thermal energy. This heats the biofluid i.e. elevates temperatures. Conversely increasing permeability parameter (fig. 5b) leads to a reduction in temperature. The increase in medium permeability implies a decrease in solid matrix fibers in the medium. This reduces the material available for thermal conduction heat transfer and manifests in a cooling of the medium. Thermal conductivity of the fluid-saturated medium is clearly influenced with a modification in permeability and as the permeability increases the heat transfer rate to the walls will increase. Increasing first and second Jeffery parameters (Figs. 5c and 5d) respectively decreases and enhances the temperature magnitudes in the medium. Retardation of the biofluid is therefore beneficial to heat transfer through the medium whereas relaxation opposes it. Fig.5e illustrates the effect of Brinkmann number on the temperature profile. Brinkman number is related to the heat conduction from the wall to the viscous fluid and characterizes the viscous dissipation term in the fluid flow. With increasing the value of Brinkman number, the thermal conductivity of the fluid decreases so greater quantity of heat can be transferred through the fluid. It is also noted that a higher temperature exists near the core region of the channel in comparison to the walls of the ciliated channel.

\section{Streamlines:}

Figs. 6-9 illustrate the influence of Hartmann number $(M)$, permeability parameter $(K)$, ratio of relaxation to retardation times $\left(\lambda_{1}\right)$ and retardation time $\left(\lambda_{2}\right)$ on the stream function. Fig. 6(a-c) show that the number of trapped boluses decreases with Hartmann number. This is due to the decelerating effect of magnetic force on velocity. It is observed from Fig. 7(a-c) that the amplitude of wave decreases with an increase in the permeability parameter since the flow is accelerated and this prevents the build-up of larger amplitudes generated by the metachronal wave motion. Fig. 8(a-c) highlight that the number of trapped boluses and their magnitudes are enhanced with 
increasing Jeffery first viscoelastic parameter i.e. with greater rheological relaxation times. The boluses are strongly stretched in the vertical direction with greater values of $\lambda_{1}$. Finally, it is evident from Fig. 9(a-c) that the size of trapped boluses is also increased with greater retardation time values $\left(\lambda_{2}\right)$.

\section{CONCLUSIONS}

A mathematical model has been presented for forced convective heat transfer in transport of an electrically-conducting viscoelastic Jeffrey physiological fluid through a ciliated channel containing a high permeability porous medium under the action of a transverse magnetic field. The flow is produced due to the metachronal wave generated by synchronized beating of cilia which follow an elliptical path. The transformed (fixed to wave frame) and non-dimensional boundary value problem (momentum and energy conservation equations and associated channel wall boundary conditions) have been solved with the semi-numerical Adomian decomposition method (ADM). Extensive details of the ADM solutions have been provided. The numerical evaluation of the power-series solutions is conducted in MATHEMATICA software. The results show that:

- Velocity field, pressure gradient and temperature profile show a similar parabolic nature and attain maximum magnitudes at the channel centre and further decrease rapidly at the walls of channel.

- The behavior of velocity field and pressure gradient is distinctly different throughout the channel.

- Velocity is decreased with increasing Hartmann number and Jeffery first viscoelastic parameter (ratio of relaxation to retardation times) in the core zone of the channel whereas the flow is accelerated near the walls.

- Velocity is elevated with increasing permeability parameter and Jeffery second viscoelastic parameter (retardation parameter) in the core zone.

- Pressure gradient is increases with higher Hartmann number whereas it is reduced with greater permeability parameter at the centre of the channel.

- Temperature profile is maximized in the core section of the channel and diminished at the walls.

- Temperature is observed to be enhanced with increasing Hartmann number and second Jeffery parameter whereas it is reduced with permeability parameter and Jeffery first 
parameter.

- There is a linear relationship between pressure rise and volume flow rate.

- Pressure rise is elevated with increasing permeability and Jeffery first parameter (relaxation to retardation time ratio) whereas it decreases with an increase in Lorentz magnetic body retarding force i.e. with greater Hartmann numbers.

- Number of trapped boluses decrease an increase in Hartmann number.

- The amplitude of streamlines decreases with an increase in permeability parameter whereas the size and the number of trapped boluses increase with greater values of Jeffery first and second viscoelastic parameters.

The current study has neglected magnetic induction and mass transfer effects which are also important in fertility devices. These aspects will be addressed in the future.

\section{REFERENCES}

[1] A.M. Mohammadi and J.L. Schroder, Laser interstitial thermal therapy in treatment of brain tumors - the NeuroBlate System, Expert Review of Medical Devices, 11, 109-119 (2014).

[2] Y. He et al, Finite element analysis of blood flow and heat transfer in an image-based human finger, Computers in Biology and Medicine, 38,_555-562 (2008).

[3] A. Bejan, Theory of heat transfer from a surface covered with hair, ASME J. Heat Transfer 112(3), 662-667 (1990).

[4] D.T. Haynie, Biological Thermodynamics, Cambridge University Press, New York (2001).

[5] A. Karampatzakis and T. Samaras Numerical model of heat transfer in the human eye with consideration of fluid dynamics of the aqueous humour, Phys. Med. Biol. 55, 5653-5678 (2010).

[6] D. Tripathi, Pandey, S.K., Bég, O. Anwar, 2013. Mathematical modelling of heat transfer effects on swallowing dynamics of viscoelastic food bolus through the human oesophagus. Int. J. Thermal Sciences, 70, 41-53 (2013).

[7] G. Horstmann, Iravani, J., Melville, G. N., and Richter, H. G., Influence of temperature and decreasing water content of inspired air on the ciliated bronchial epithelium, Acta Oto-Laryngol. 84, 124-131 (1977).

[8] A. Zaman, N. Ali, O. Anwar Bég and M. Sajid, Heat and mass transfer to blood flowing through a tapered overlapping stenosed artery, Int. J. Heat Mass Transfer, 95, 1084-1095 (2015). 
[9] D. Sompel et al., Modeling of experimentally created partial-thickness human skin burns and subsequent therapeutic cooling: a new measure for cooling effectiveness, J. Med. Eng. Phys., 31, 624-31 (2009).

[10] S.C. Jiang et al., Effects of thermal properties and geometrical dimensions on skin burn injuries, Burns, 28, 713-717 (2002).

[11] M. J. Uddin, O. Anwar Bég and M.N. Kabir, Computational investigation of Stefan blowing and multiple slip effects on buoyancy-driven bioconvection nanofluid flow with micro-organisms, Int. J. Heat Mass Transfer, 95, 116-130 (2016).

[12] Assma F. Elsayed and O. Anwar Bég, New computational approaches for biophysical heat transfer in tissue under ultrasonic waves: Variational iteration and Chebyschev spectral simulations, J. Mechanics Medicine Biology, 14, 3, 1450043.1-1450043.17 (17 pages) (2014).

[13] Prek, M., 2006. Thermodynamical analysis of human thermal comfort. Energy, 31, 732-743.

[14] K. Diller KR, Valvano JW, Pearce JA. Bioheat Transfer. In: Kneith F, editor. CRC Handbook of Heat Transfer, 2nd ed. 2005.

[15] Eberhart, R.C., Shitzer, A.(editors), Heat Transfer in Medicine and Biology: Analysis and Applications (Vol. 2). Springer Science \& Business Media (2012).

[16] A. V. Karpov, Mathematical Modeling in Medicine, Encyclopedia of Life Support Systems (EOLSS), New York, USA (2015).

[17] T. Togawa et al., Observation of blood flow E.M.F. in externally applied strong magnetic field by surface electrodes, Med. Biol. Eng., 5, 169-170 (1967).

[18] G. Saygih, Aydinlik E, Ercan MT, et al., Investigation of the effect of magnetic retention systems used in prostheses on buccal mucosal blood flow. Int J Prosthodontics, 5, 326-363 (1992).

[19] G.M. Nijm et al., Extraction of the magnetohydrodynamic blood flow potential from the surface electrocardiogram in magnetic resonance imaging, Medical \& Biological Engineering \& Computing, 46, 729-733 (2008).

[20] N. Mustapha et al., Unsteady magnetohydrodynamic blood flow through irregular multistenosed arteries, Computers in Biology and Medicine, 39, 896-906 (2009).

[21] C. Valbona, Hazelwood CF, Jurida G., Response of pain to static magnetic fields in postpolio patients: a double-blind study. Arch Phys Med Rehabil., 78, 1200-1203 (1997).

[22] D. Tripathi and O. Anwar Bég, A study of unsteady physiological magneto-fluid flow and heat transfer through a finite length channel by peristaltic pumping, Proceedings of the Institution of Mechanical Engineers, Part H-J. Engineering in Medicine, 226, 631 - 644 (2012). 
[23] S. Kenjereš and Righolt, B.W., Simulations of magnetic capturing of drug carriers in the brain vascular system. Int. J. Heat and Fluid Flow, 35, 68-75 (2012).

[24] M.M. Rashidi, M. Keimanesh, O. Anwar Bég and T.K. Hung, Magneto-hydrodynamic biorheological transport phenomena in a porous medium: A simulation of magnetic blood flow control and filtration, International Journal for Numerical Methods in Biomedical Engineering, 27, 805-821 (2011).

[25] D. Tripathi, R. Jhorar, O. Anwar Bég and A. Kadir, Electro-magneto-hydrodynamic peristaltic pumping of couple stress biofluids through a complex wavy micro-channel, J. Molecular Liquids, 236, 358-367 (2017).

[26] A.L. Copley, Fluid mechanics and biorheology, Thrombosis Research, 57, 315-331 (1990).

[27] S.M.Mitran, A numerical model of viscoelastic layer entrainment by airflow in cough, $15^{\text {th }}$ International Congress on Rheology, Monterey, CA, USA, August $3^{\text {rd }}-8^{\text {th }}$ (2008).

[28] P.A.Vasquez et al. Modeling and simulation of mucus flow in human bronchial epithelial cell cultures-Part I: Idealized axisymmetric swirling flow,_PLoS Comput Biol., 12(8):e1004872 (2016).

[29] G. Arwatz and A.J. Smits, A viscoelastic model of shear-induced hemolysis in laminar flow, Biorheology, 50, 45-55 (2013).

[30] K. Ishimoto et al., Human sperm swimming in a high viscosity mucus analogue, J. Theoretical Biology, 446, 1-10 (2018).

[31] V. Ribitsch et al., Viscoelasticity of synovial fluids and hyaluronic acid, Biorheology, 32, 209-220 (1995).

[32] M.D. Hugo et al., Rheumatoid hyper-viscosity syndrome, The American Journal of Medicine, 49, 484-493 (1970).

[32] Y. Hayashi, Fluid-dynamical study of protoplasmic streaming in a plant cell,_J. Theoretical Biology, 85, 451-467 (1980).

[34] D. Oriola, H. Gadêlha, J. Casademunt, Nonlinear amplitude dynamics in flagellar beating, $R$. Soc. Open Sci., 4 (3) (2017).

[35] C.B. Lindemann, K.A. Leisch, Flagellar and ciliary beating: the proven and the possible, $J$. Cell Sci., 123, 519-528 (2010).

[36] C.B. Lindemann, A model of flagellar and ciliary functioning which uses the forces transverse to the axoneme as the regulator of dynein activation, Cell Motil. Cytoskelton, 29, 141-154 (1994).

[37] A.M. Siddiqui, Haroon, T., Rani, M., Ansari, A.R., An analysis of the flow of a power law 
fluid due to ciliary motion in an infinite channel, Biorheology, 24, 56-69 (2010).

[38] K. Maqbool, Mann, A.B., Siddiqui, A.M., Shaheen, S., Fractional generalized Burgers' fluid flow due to metachronal waves of cilia in an inclined tube. Adv. Mech. Eng., 9,.1687814017715565 (2017).

[39] A.M. Siddiqui., Farooq, A.A., Rana, M.A., An investigation of non-Newtonian fluid flow due to metachronal beating of cilia in a tube. Int. J.Biomathatics, 8, p.1550016 (2015).

[40] N.S. Akbar, Tripathi, D., Bég, O. Anwar, Khan, Z.H., MHD dissipative flow and heat transfer of Casson fluids due to metachronal wave propulsion of beating cilia with thermal and velocity slip effects under an oblique magnetic field. Acta Astronautica, 128, 1-12 (2016).

[41] A. Shaheen and S. Nadeem, Metachronal wave analysis for non-Newtonian fluid under thermophoresis and Brownian motion effects. Results in Physics, 7, 2950-2957 (2017).

[42] R.E. Abo-Elkhair, Mekheimer, K.S., Moawad, A.M.A., Cilia walls influence on peristaltically induced motion of magneto-fluid through a porous medium at moderate Reynolds number: numerical study. J. Egyptian Mathematical Society, 25, 238-251 (2017).

[43] M.D.C. Lopez-Garcia, R.L. Monson, K. Haubert, M.B. Wheeler, D.J. Beebe, Sperm motion in a microfluidic fertilization device, Biomed. Microdevices, 10, 709-718 (2008).

[44] N. Hosomura et al., A case of foregut gastric duplication cyst with pseudostratified columnar ciliated epithelium, Clinical Journal of Gastroenterology, 5,_82-87 (2012).

[45] V. Ramachandra Prasad, S. Abdul Gaffar, E. Keshava Reddy, and O. Anwar Bég, Flow and heat transfer of Jefferys non-Newtonian fluid from a horizontal circular cylinder, AIAA $J$. Thermophysics and Heat Transfer, 28, 764-770 (2014).

[46] G. Adomian, Solving Frontier Problems of Physics: The Decomposition Method, Kluwer, Boston, USA (1994).

[47] O. Anwar Bég, D. Tripathi, T. Sochi and PK Gupta, Adomian decomposition method (ADM) simulation of magneto-biotribological squeeze film with magnetic induction effects, J. Mechanics Medicine Biology, 15, 1550072.1-1550072.23 (2015).

[48] O. Anwar Bég, Multi-physical computational modelling of nanofluid bioconvection flows, $A$. Sohail, Z. Li (Eds.): Computational Approaches in Biomedical Nano-Engineering, Wiley-CVH, China, Chapter 5, 100-130 (2019). In press

[49] O. Anwar Bég, F. Mabood and M. Nazrul Islam, Homotopy simulation of nonlinear unsteady rotating nanofluid flow from a spinning body, Int. J. Engineering Mathematics, Volume 2015, Article ID 272079, 15 pages (2015). 
[50] G. C. Schit, N. K. Ranjit and A. Sinha, Adomian decomposition method for magnetohydrodynamic flow of blood induced by peristaltic waves, J. Mechanics Medicine Biology, 17, 1750007 (28 pages) (2017).

[51] G.B. Thurston and H. Greiling, Viscoelastic properties of pathological synovial fluids for a wide range of oscillatory shear rates and frequencies, Rheol. Acta, 17, 433-445 (1978).

\section{APPENDIX - ADOMIAN SOLUTIONS}

$$
\begin{aligned}
& A_{1}(x)=\frac{1}{1008000 h}(1512000(-1+\bar{Q})+h(-504000 u(h)+A h(25200 \\
& \left.\left.\left.+A h^{2}\left(-1320+67 A h^{2}\right)\right)(1-\bar{Q}+h u(h))\right)\right)+\frac{1}{84000 h^{3}} \beta \lambda_{2}((15(2520 \\
& \left.+A h^{2}\left(-228+5 A h^{2}\right)\right)(-1+\bar{Q})^{2}-9 h\left(7000+3 A h^{2}\left(-180+7 A h^{2}\right)\right)(-1+\bar{Q}) u(h) \\
& \left.+2 h^{2}\left(12600+A h^{2}\left(-1770+167 A h^{2}\right)\right) u^{2}(h)\right) h^{\prime}+h^{2}(12600(-1+\bar{Q}-h u(h)) \\
& \left.\left.-60 A h^{2}(-33+33 \bar{Q}+2 h u(h))+A^{2} h^{4}(181(-1+\bar{Q})+39 h u(h))\right) u^{\prime}(h)\right),
\end{aligned}
$$

$$
\begin{aligned}
A_{2}(x)= & -\frac{1}{756000 h^{3}}\left(-378000+A h^{2}\left(37800+A h^{2}\left(-2430+133 A h^{2}\right)\right)\right) \\
& \times(1-\bar{Q}+h u(h))-\frac{1}{21000 h^{5}} \beta \lambda_{2}\left(\left(15\left(-15+A h^{2}\right)\left(-84+5 A h^{2}\right)\right.\right. \\
& \times(-1+\bar{Q})^{2}-h\left(31500+A h^{2}\left(-3555+167 A h^{2}\right)\right)(-1+\bar{Q}) u(h) \\
& \left.+h^{2}\left(12600+A h^{2}\left(-2220+227 A h^{2}\right)\right) u^{2}(h)\right) h^{\prime}+h^{2}((6300 \\
& \left.+A h^{2}\left(-1215+118 A h^{2}\right)\right)(-1+\bar{Q})+h\left(-15+A h^{2}\right) \\
& \left.\left.\left.\times\left(420+17 A h^{2}\right) u(h)\right) u^{\prime}(h)\right)\right),
\end{aligned}
$$

$$
\begin{aligned}
A_{3}(x)= & \frac{1}{56000 h^{3}} A\left(1400+A h^{2}\left(-140+9 A h^{2}\right)\right)(1-\bar{Q}+h u(h)) \\
& -\frac{1}{14000 h^{7}} \beta \lambda_{2}\left(\left(-15\left(420+A h^{2}\left(-98+5 A h^{2}\right)\right)(-1+\bar{Q})^{2}\right.\right. \\
& +h\left(10500+A h^{2}\left(-2310+149 A h^{2}\right)\right)(-1+\bar{Q}) u(h)-2 h^{2}(2100 \\
& \left.\left.+A h^{2}\left(-595+72 A h^{2}\right)\right) u^{2}(h)\right) h^{\prime}+h^{2}\left(A^{2} h^{4}(71-71 \bar{Q}+h u(h))\right. \\
& \left.\left.\left.+2100(1-\bar{Q}+h u(h))-70 A h^{2}(9-9 \bar{Q}+4 h u(h))\right) u^{\prime}(h)\right)\right),
\end{aligned}
$$




$$
\begin{aligned}
A_{4}(x)= & -\frac{1}{16800 h^{3}} A^{2}\left(-10+A h^{2}\right)(1-\bar{Q}+h u(h)) \\
& -\frac{1}{4200 h^{7}} A \beta \lambda_{2}\left(\left(15\left(-9+A h^{2}\right)(-1+\bar{Q})^{2}\right.\right. \\
& -9 h\left(-25+3 A h^{2}\right)(-1+\bar{Q}) u(h) \\
& \left.+h^{2}\left(-90+17 A h^{2}\right) u^{2}(h)\right) h^{\prime}+h^{2}\left(A h^{2}(-8\right. \\
& \left.+8 \bar{Q}-3 h u(h)]+45(1-\bar{Q}+h u(h))) u^{\prime}(h)\right),
\end{aligned}
$$

$$
\begin{aligned}
A_{5}(x)= & \frac{1}{120960 h^{3}} A^{3}(1-\bar{Q}+h u(h))-\frac{1}{3360 h^{7}}\left(A^{2}(1-\bar{Q}\right. \\
& \left.+h u(h)) \beta \lambda_{2}\left((-3+3 \bar{Q}-2 h u(h)) h^{\prime}+h^{2} u^{\prime}(h)\right)\right),
\end{aligned}
$$

$$
\begin{aligned}
B_{1}(x)= & -\frac{1}{10710470950058880000000000 h^{4}\left(1+\lambda_{1}\right)}\left[B r \left(21420941900117760000000000 h^{6}\right.\right. \\
& +(1-\bar{Q}+h u(h)) \beta \lambda_{2}\left(\left(A^{9} h^{18}(10233330719421(-1+\bar{Q})\right.\right. \\
& -13415170239448 h u(h))(-1+\bar{Q}-h u(h))-1486674000 A^{6} h^{12}(7370113(-1+\bar{Q}) \\
& -19503891 h u(h))(-1+\bar{Q}-h u(h))-1159141877712000000 A^{3} h^{6}(1-\bar{Q} \\
& +h u(h))(256-256 \bar{Q}+255 h u(h))+3213141285017664000000000\left(9(-1+\bar{Q})^{2}\right. \\
& \left.\left.-5 h(-1+\bar{Q}) u(h)+h^{2} u^{2}(h)\right)\right) h^{\prime}+7 h^{2}\left(165591696816000000 A^{3} h^{6}(1-\bar{Q}+h u(h))\right. \\
& -2576996039196000 A^{6} h^{12}(1-\bar{Q}+h u(h))+454548502861 A^{9} h^{18}(1-\bar{Q}+h u(h)) \\
& \left.\left.+459020183573952000000000(-3+3 \bar{Q}+2 h u(h))) u^{\prime}(h)\right)\right],
\end{aligned}
$$

$$
\begin{aligned}
B_{2}[x]= & \frac{1}{48009024000000000 h^{8}\left(1+\lambda_{1}\right)}[B r(-1+\bar{Q}-h u) \\
& \times \beta \lambda_{2}\left(\left(36006768000000 A^{3} h^{6}(1-\bar{Q}+h u(h))^{2}\right.\right. \\
& -7794468000 A^{6} h^{12}(1-\bar{Q}+h u(h))^{2}+1185163 A^{9} h^{18}(1-\bar{Q}+h u(h))^{2} \\
& \left.-72013536000000000\left(3+3 \bar{Q}^{2}-3 \bar{Q}(2+h u(h))+h u(h)(3+h u)\right)\right) h^{\prime} \\
& \left.-72013536000000000 h^{2}(-1+\bar{Q}) u^{\prime}(h)\right],
\end{aligned}
$$




$$
\begin{aligned}
& B_{3}(x)=\frac{1}{360067680000000000 h^{10}\left(1+\lambda_{1}\right)}\left[B r(1-\bar{Q}+h u(h))^{2} \beta \lambda_{2}\right. \\
& \times((-216040608000000000(3-3 \bar{Q}+2 h u(h)) \\
& +36006768000000 A^{3} h^{6}(26-26 \bar{Q}+25 h u(h)) \\
& -78732000 A^{6} h^{12}(-3891(-1+\bar{Q})+3932 h u(h)) \\
& \left.+133 A^{9} h^{18}(-438972(-1+\bar{Q})+449621 h u(h))\right) h^{\prime} \\
& +h^{2}\left(216040608000000000-36006768000000 A^{3} h^{6}\right. \\
& \left.\left.-3228012000 A^{6} h^{12}+1416317 A^{9} h^{18}\right) u^{\prime}(h)\right]
\end{aligned}
$$

$$
\begin{aligned}
& B_{4}(x)=\frac{1}{24893568000000000 h^{6}\left(1+\lambda_{1}\right)}\left[A^{3} B r(1-\bar{Q}+h u(h))^{2} \beta \lambda_{2}\right. \\
& \times\left(\left(A^{6} h^{12}(10685101(-1+\bar{Q})-10831802 h u(h))\right.\right. \\
& -444528000000(204-204 \bar{Q}+181 h u(h)) \\
& +5292000 A^{3} h^{6}(-8767(-1+\bar{Q}+8625 h u(h))) h^{\prime} \\
& \left.+h^{2}\left(10224144000000-751464000 A^{3} h^{6}-146701 A^{6} h^{12}\right) u^{\prime}(h)\right],
\end{aligned}
$$

$$
\begin{aligned}
B_{5}(x)= & \frac{1}{6001128000000000 h^{8}\left(1+\lambda_{1}\right)}\left[A^{3} B r(1-\bar{Q}+h u(h))_{2}^{2} \beta \lambda\right. \\
& \times((1000188000000(14-14 \bar{Q}+11 h u(h)) \\
& -81000 A^{3} h^{6}(-165937(-1+\bar{Q})+156106 h u(h)) \\
& \left.+7 A^{6} h^{12}(-558740(-1+\bar{Q})+554657 h u(h))\right) h^{\prime} \\
& \left.\left.-3 h^{2}\left(1000188000000-265437000 A^{3} h^{6}+9527 A^{6} h^{12}\right) u^{\prime}(h)\right)\right],
\end{aligned}
$$

$$
\begin{aligned}
& B_{6}(x)=\frac{1}{158429779200000000 h^{10}\left(1+\lambda_{1}\right)}\left[A^{3} B r(1-\bar{Q}+h u(h))^{2} \beta \lambda_{2}\right. \\
& \times\left(\left(A^{6} h^{12}(101393121(-1+\bar{Q})-98073227 h u(h))\right.\right. \\
& -30005640000000(3-3 \bar{Q}+2 h u(h)) \\
& \left.+10206000 A^{3} h^{6}(-24819(-1+\bar{Q})+22055 h u(h))\right) h^{\prime}+2 h^{2}(15002820000000 \\
& \left.\left.\left.-14104692000 A^{3} h^{6}+1659947 A^{6} h^{12}\right) u^{\prime}(h)\right)\right]
\end{aligned}
$$




$$
\begin{aligned}
B_{7}(x)= & \frac{1}{107872128000000 h^{6}\left(1+\lambda_{1}\right)}\left[A^{6} B r(1-\bar{Q}+h u(h))^{2} \beta \lambda_{2}\right. \\
& \times((-25200(-2893(-1+\bar{Q})+2400 h u(h)) \\
& \left.+A^{3} h^{6}(-45924(-1+\bar{Q})+43127 h u(h))\right) h^{\prime} \\
& \left.\left.+h^{2}\left(12423600-2797 A^{3} h^{6}\right) u^{\prime}(h)\right)\right]
\end{aligned}
$$

$$
\begin{aligned}
B_{8}(x)= & \frac{1}{426746880000000 h^{8}\left(1+\lambda_{1}\right)}\left[A^{6} B r(1-\bar{Q}+h u(h))^{2} \beta \lambda_{2}\right. \\
& \times\left(\left(A^{3} h^{6}(82529(-1+\bar{Q})-74860 h u(h))\right.\right. \\
& +2646000(25-25 \bar{Q}+19 h u(h))) h^{\prime} \\
& \left.\left.+h^{2}\left(-15876000+7669 A^{3} h^{6}\right) u^{\prime}(h)\right)\right]
\end{aligned}
$$

$$
\begin{aligned}
B_{9}(x)= & \frac{1}{2448460224000000 h^{10}\left(1+\lambda_{1}\right)}\left[A^{6} B r(1-\bar{Q}+h u(h))^{2} \beta \lambda_{2}\right. \\
& \times\left(\left(-11907000(3-3 \bar{Q}+2 h u(h))+A^{3} h^{6}(-144084(-1+\bar{Q})\right.\right. \\
& \left.\left.+125069 h u(h))) h^{\prime}+5 h^{2}\left(2381400-3803 A^{3} h^{6}\right) u^{\prime}(h)\right)\right],
\end{aligned}
$$

$$
\begin{aligned}
B_{10}(x)= & \frac{1}{90091008000000 h^{6}\left(1+\lambda_{1}\right)}\left[A^{9} B r(1-\bar{Q}+h u(h))^{2}\right. \\
& \left.\times \beta \lambda_{2}\left((1029(-1+\bar{Q})-841 h u(h)) h^{\prime}+188 h^{2} u^{\prime}(h)\right)\right], \\
B_{11}(x)= & -\frac{1}{3129477120000 h^{8}\left(1+\lambda_{1}\right)}\left[A^{9} B r(1-\bar{Q}+h u(h))^{2}\right. \\
& \left.\times \beta \lambda_{2}\left((-4+4 \bar{Q}-3 h u(h)) h^{\prime}+h^{2} u^{\prime}(h)\right)\right]
\end{aligned}
$$

$$
\begin{aligned}
B_{12}(x)= & \frac{1}{47112855552000 h^{10}\left(1+\lambda_{1}\right)}\left[A^{9} B r(1-\bar{Q}+h u(h))^{2}\right. \\
& \left.\times \beta \lambda_{2}\left((-3+3 \bar{Q}-2 h u(h)) h^{\prime}+h^{2} u^{\prime}(h)\right)\right] .
\end{aligned}
$$

